\title{
Propuesta de rúbrica para coevaluación de trabajos en asignaturas de máster
}

\author{
Juan V. Oltra Gutiérrez ${ }^{\text {a }}$, Yeamduan Narangajavana-Kaosiri ${ }^{\text {b }}$ \\ ${ }^{a}$ Departamento de Organización de Empresas, Universitat Politècnica de València. Valencia. \\ jvoltra@omp.upv.es \\ ${ }^{b}$ Departamento de Administración de empresas y Marketing, Universitat Jaume I, Castellon, España \\ ynaranga@uji.es
}

\begin{abstract}
Resumen
El presente trabajo presenta una propuesta para coevaluar trabajos mediante rúbrica. Se abordan en él los principales problemas que el docente debe afrontar y las soluciones propuestas.

La propuesta se muestra adaptada a una asignatura de corte tecnológico, con un peso muy concreto en la evaluación final, donde estos extremos, los más fáciles de adaptar, muestran al tiempo que la singularidad de la experiencia no lo es tal por la procedencia de los alumnos.
\end{abstract}

Palabras clave: Rúbricas, Coevaluación, Innovación Docente.

\section{Introducción}

Todo docente se encuentra con una serie de limitaciones, siendo de las más comunes el escaso tiempo y la resistencia al cambio ante los cambios que las nuevas metodologías docentes imponen. (Viñas Pérez, 2015) (Viñao, 2006). Por otra parte, cuando se trata de estudios más allá del grado, de master o de tercer ciclo, entre los alumnos hay una casuística inmensa de la que suele destacar dos factores: la diferente titulación de partida y la mayor maduración intelectual del alumno frente a etapas anteriores (Peña, Gadañón, Crespo, \& Sánchez, 2012) (Oltra Gutiérrez, Juan Vicente, 2018)

Con la siguiente propuesta tratamos de apoyarnos en esa distinta casuística para paliar las limitaciones docentes. La idea es hacer que "trabajen ellos", como método, por una parte, que apoya su crecimiento intelectual, y por otra, rebaja la carga de corrección del profesor al facilitar un pre-filtrado de los trabajos a corregir, cada vez más con las llamadas metodologías activas. (Gallego Arrufat \& Raposo Rivas, 2014) 


\section{Punto de partida}

En el plan de estudios del Master Universitario de Gestión de la Información se reciben alumnos de múltiples procedencias: Grado de Informática, Derecho, Periodismo, Ingeniería Industrial, Telecomunicaciones... gente de procedencia muy diversa no solo en titulaciones sino en nacionalidad (Rusia, Ucrania, Brasil, Colombia, Cuba, España, etc.) No se puede establecer un hilo común más allá del que da que se trate de personas en un momento vital que les ha llevado en su inmensa mayoría (la última encuesta realizada daba cifras superiores al 75\%) trabajan mientras cursan los estudios.

Esa diversidad provoca que se deban buscar caminos comunes. Para ello, se disponen los trabajos de la asignatura en forma de entrega por grupos, para que exista la tan necesaria transfusión de conocimientos de unos a otros y, por otra parte, faciliten la corrección al docente, al reducirse el número de entregas y ser estas, obligadas a cubrir una serie de aspectos, homogéneas en su forma.

Esta diversidad afecta sobre todo a la asignatura MLD: Marco Legal y Deontológico de la información (Oltra Gutiérrez, 2019), pues el conocimiento de partida de la legislación Española y europea probablemente sea el más heterogéneo de todos los que conforman las distintas facetas de ese prisma que es el master.

Para reforzar ese intercambio no solo se realizan los trabajos en grupo, sino que antes de ser entregados al profesor, estos son coevaluados entre sí, por otros grupos de alumnos.

Eso permite una visión mayor de los conocimientos que realmente comparten y, por otra, sirve de criba para el docente, pues los que tienen una calificación muy baja son revisados de nuevo por los autores, evitando que sea el profesor quien les da esa vuelta de tuerca.

Para dar uniformidad y facilitar el trabajo (Ponce Morales \& Rodríguez Mendoza, 2013) (Martínez-Azúa, Sandía, Rey, \& Dios, 2016), se propone una rúbrica.

\section{Pruebas a evaluar}

Siguiendo la guía docente, encontramos una serie de pruebas, que presentamos en la siguiente tabla.

Anticipemos que esos tres puntos, aun en distintos epígrafes, se corresponden al trabajo de la asignatura, en sus distintas fases. Estos tres puntos son realizados, como se ha anticipado, 
en grupo, siendo divididos en cuatro bloques homogéneos en su nota de 0,75 puntos cada uno.

Tabla 1. Elementos a evaluar.

\begin{tabular}{|c|c|c|c|}
\hline & $\mathbf{N}^{o}$ entregas & Peso $(\%)$ & Puntos \\
\hline Examen oral. & 1 & 7,5 & 0,75 \\
\hline Mapa conceptual & 1 & 7,5 & 0,75 \\
\hline Trabajo académico & 1 & 7,5 & 0,75 \\
\hline Coevaluación & 1 & 7,5 & 0,75 \\
\hline Caso & 7 & 70 & 7 \\
\hline TOTAL & & 100 & 10 \\
\hline
\end{tabular}

El trabajo en sí consiste en la creación de un mapa conceptual sobre un asunto de interés para la asignatura, que ha de ir acompañado de una memoria descriptiva, de entre dos y cinco páginas.

¿Cuál es la división del trabajo? Veámosla repartida en el tiempo.

En una primera entrega, como borrador, se entrega la memoria descriptiva, que apuntará cómo será el mapa conceptual final. Esta entrega es la que se coevalúa y la que veremos con detalle a continuación. El peso de esta prueba será de 0,75 puntos. Hay que indicar que la nota en este caso no va asociada a la calidad de la entrega de cada grupo, sino a la calidad de las correcciones de los mismos por parte de otros grupos.

Una segunda entrega lleva dos partes asociadas: esa misma memoria descriptiva, ya en fase final, y el mapa conceptual en sí. Cada una de esas dos partes recibe una calificación máxima de 0,75 puntos.

Una tercera fase pasa por la presentación oral del mapa conceptual, de forma que al finalizar la misma, deben responder unas preguntas por parte del docente. Esta parte recibe así mismo una calificación de 0,75 puntos.

\section{Rúbrica propuesta}

La creación de una rúbrica es una de las más intensas que un docente puede realizar: esta debe ser legible por todos, debe decir exactamente lo que se pretende, ser clara y sin 
ambigüedades y, además, adaptable según las circunstancias. (Picornell Lucas, 2014) (Chica Merino, 2011)

En el caso que nos ocupa, un borrador de poco tamaño, documento además asociado a un mapa conceptual y con una nota reducida $(0,75$ puntos) se propone una rúbrica de sencilla y rápida aplicación, basada en la simple comprobación de una serie de elementos distribuidos en tres categorías: elementos principales del mapa conceptual, relaciones principales del mapa conceptual y bibliografía y otros aspectos formales.

Cada una de esas tres categorías puede recibir una nota que va del rango de 0 a 0,25 puntos si se cumplen las condiciones asociadas (ver tabla 2). Para los aspectos formales y bibliográficos se ha preparado una lista breve de aspectos a considerar de forma que la nota será proporcional a la presencia (o ausencia) de los mismos.

Los elementos a revisar en esta última categoría serían:

1. El documento no presenta problemas de legibilidad

2. Es correcto sintáctica y semánticamente

3. La bibliografía es adecuada extensión (mínimo, cuatro fuentes)

4. La bibliografía es adecuada en calidad (fuentes procedentes de artículos de investigación, libros... no Wikipedia o similar)

5. La bibliografía y está bien referenciada (emplea alguna norma como IEEE, APA o MLA)

Hay que remarcar que la nota que los alumnos indican no será la asociada al grupo que corrijan. Sirve para determinar su propia nota: de qué manera han corregido. Para esto será preciso que cada grupo evaluado indique (con una o dos líneas, no hace falta más) el porqué de cada decisión en la coevaluación.

Para el docente sirve además para establecer una retroalimentación a los alumnos sobre cómo va su propio trabajo. Esto agiliza mucho la tarea del docente, que evita una precorrección y, además, se asegura de mejorar el producto final del trabajo de sus alumnos, pues estos han corregido, al menos en los aspectos formales, aquellos aspectos con problemas.

Cabe añadir una nota un tanto subjetiva: a lo largo de los años que se ha venido experimentando en la asignatura con las técnicas de coevaluación, la nota que los alumnos se dan a sí mismos suelen ser mucho más bajas que las que el docente les daría. Esto es: sus criterios de corrección suelen ser mucho más duros. 
Tabla 2. Rúbrica

\begin{tabular}{|c|c|c|c|}
\hline Elemento & $\begin{array}{l}\text { Elemento principal: } \\
\text { razón de su presencia }\end{array}$ & $\begin{array}{l}\text { Elemento principal: } \\
\text { Relaciones con los } \\
\text { otros elementos que se } \\
\text { señalan }\end{array}$ & $\begin{array}{l}\text { Bibliografia } \mathbf{y} \\
\text { aspectos } \\
\text { formales }\end{array}$ \\
\hline 0 puntos & No aparece ninguno- & $\begin{array}{l}\text { No se indica relación } \\
\text { alguna }\end{array}$ & $\begin{array}{lr}\text { Carece } & \text { de } \\
\text { bibliografía. } & \\
\text { Párrafos } & \\
\text { ilegibles } & \text { o } \\
\text { documento mal } \\
\text { estructurado }\end{array}$ \\
\hline 0,05 puntos & $\begin{array}{l}\text { Aparece solo un elemento, } \\
\text { y no se explica nada sobre } \\
\text { él. }\end{array}$ & $\begin{array}{l}\text { Se indica alguna } \\
\text { relación, pero no otras, } \\
\text { y no se explican }\end{array}$ & $\begin{array}{l}\text { Cumple solo } \\
\text { con uno de los } \\
\text { elementos } \\
\text { revisar. }\end{array}$ \\
\hline 0,1 puntos & $\begin{array}{l}\text { Aparecen de } 2 \text { a } 5 \\
\text { elementos y al menos de } \\
\text { uno no se explica nada. }\end{array}$ & $\begin{array}{l}\text { Se indican las } \\
\text { relaciones entre los } \\
\text { elementos indicados, } \\
\text { pero no se explican. }\end{array}$ & $\begin{array}{l}\text { Cumple con dos } \\
\text { de los elementos } \\
\text { a revisar. }\end{array}$ \\
\hline 0,15 puntos & $\begin{array}{l}\text { Aparecen más de } 5 \\
\text { elementos, aunque algunos } \\
\text { carecen de explicación. }\end{array}$ & $\begin{array}{l}\text { Se indican algunas } \\
\text { relaciones, algunas se } \\
\text { explica, otras no. }\end{array}$ & $\begin{array}{l}\text { Cumple con tres } \\
\text { de los elementos } \\
\text { a revisar. }\end{array}$ \\
\hline 0,20 puntos & $\begin{array}{l}\text { Aparecen más de cinco } \\
\text { elementos, } \\
\text { subelementos, aunque la } \\
\text { aparición de algunos } \\
\text { carece de explicación. }\end{array}$ & $\begin{array}{l}\text { Se indican todas las } \\
\text { relaciones, pero alguna } \\
\text { no se explica. }\end{array}$ & $\begin{array}{lr}\text { Cumple } & \text { con } \\
\text { cuatro de } & \text { los } \\
\text { elementos } & \text { a } \\
\text { revisar. } & \end{array}$ \\
\hline $\begin{array}{l}0,25 \text { puntos } \\
\text { (máximo }\end{array}$ & $\begin{array}{llr}\text { Aparecen más de cinco } \\
\text { elementos, } & & \text { con } \\
\text { subelementos, } & \text { y } & \text { los } \\
\text { elementos } & \text { aparecen } \\
\text { explicados. } & & \end{array}$ & $\begin{array}{l}\text { Se indican y explican } \\
\text { todas las relaciones. }\end{array}$ & $\begin{array}{l}\text { Cumple todos } \\
\text { los elementos a } \\
\text { revisar. }\end{array}$ \\
\hline
\end{tabular}

Fuente: elaboración propia 


\section{Conclusiones}

En esta experiencia aunamos el uso de rúbricas, que permite una mayor claridad al alumno de cara a la eterna pregunta "¿qué espera de mí el profesor?” con la coevaluación, que permite una visión general, de forma que viendo los errores en el trabajo ajeno no solo se detectan mejor los propios, sino que con la necesaria retroalimentación, al ser corregidos por sus iguales, los alumnos tiene la percepción de que sus errores no han sido detectados por el ojo exquisito de un profesor, sino porque son evidentes.

Además, al formar grupos con alumnos de distinta procedencia, la tutorización inter-pares se enriquece $\mathrm{y}$, aprovechando su mayor maduración al ser alumnos con una edad mayor y obligaciones vitales más intensas que las de los alumnos medios de grado, el aprendizaje se refuerza.

\section{Referencias}

Chica Merino, E. (2011). Una propuesta de evaluación para el trabajo en grupo mediante rúbrica. EA, Escuela abierta: revista de Investigación Educativa , 67-82.

Gallego Arrufat, M., \& Raposo Rivas, M. (2014). Compromiso del estudiante y percepción del proceso evaluador basado en rúbricas. REDU: Revista de Docencia Universitaria, 197-215.

Martínez-Azúa, B., Sandía, M., Rey, M., \& Dios, N. (2016). Aprendizaje cooperativo interdisciplinar y rúbricas para la mejora del proceso de enseñanza-aprendizaje. @ tic. revista d'innovació educativa, 10-19.

Oltra Gutiérrez, J. V. (1 de marzo de 2019). Marco Legal y Deontológico de la Información. Obtenido de Master MUG: https://www.upv.es/pls/oalu/sic_asi.Busca_Asi?p_codi=34015\&p_caca=2019\&P_IDIOMA=c\&p_ vista=MSE\&p_tit=2255

Oltra Gutiérrez, Juan Vicente. (2018). Gamificación e Informática. Comparativa de una experiencia mixta en Grado y Máster. IN-RED 2018. IV Congreso Nacional de Innovación Educativa y Docencia en Red (págs. 480-491). València: Editorial Universitat Politècnica de València.

Peña, C., Gadañón, M., Crespo, J., \& Sánchez, R. (2012). Evaluación y adaptación de las metodologías docentes en las enseñanzas de máster. X Jornades de Xarxes d'Investigació en Docència Universitària: la participació i el compromís de la comunitat universitària (págs. 875886). Barcelona: Instituto de Ciencias de la Educación.

Picornell Lucas, A. (2014). La coevaluación de competencias en el Grado en Trabajo Social mediante el uso de la rúbrica. AZARBE, Revista Internacional de Trabajo Social y Bienestar. 
Ponce Morales, A., \& Rodríguez Mendoza, N. (2013). El trabajo colaborativo a distancia y el proceso de autoevaluación y coevaluación mediante rúbricas. Memorias del XXI Encuentro Internacional de Educación a Distancia . México: UDGVirtual.

Viñao, A. (2006). El éxito o fracaso de las reformas educativas: condicionantes, limitaciones, posibilidades. En J. Gimeno, La reforma necesaria: Entre la política educativa y la práctica escolar (págs. 43-60). Santander: Gobierno de Cantabria, Consejería de Educación.

Viñas Pérez, G. (2015). Los métodos participativos en una enseñanza desarrolladora. Posibles soluciones a sus limitaciones. Revista Cubana de Educación Superior, 77-87. 